\title{
Decomposição e Atividade Microbiana da Serapilheira em Coberturas Florestais no Sul do Espírito Santo
}

\author{
Marilia Alves Grugiki ${ }^{1}$, Felipe Vaz Andrade ${ }^{2}$, Renato Ribeiro Passos ${ }^{2}$, \\ Anna Carolyna Fernandes Ferreira ${ }^{3}$
}

${ }^{1}$ Programa de Pós-graduação em Ciências Florestais, Universidade Federal Rural de Pernambuco - UFRPE, Recife/PE, Brasil

${ }^{2}$ Departamento de Produção Vegetal, Universidade Federal do Espirito Santo - UFES, Alegre, ES/Brasil

${ }^{3}$ Programa de Pós-graduação em Produção Vegetal, Universidade Federal do Espirito Santo - UFES, Alegre/ES, Brasil

\begin{abstract}
RESUMO
O processo de decomposição de serapilheira deve ser amplamente estudado por se tratar de um fator-chave na manutenção e ciclagem de nutrientes nos ecossistemas. Este estudo teve como objetivos: avaliar a decomposição, a liberação de nutrientes e a atividade microbiana da serapilheira em coberturas florestais de floresta secundária, Acacia mangium, Sapindus saponaria e Hevea brasiliensis, no sul do Espírito Santo. A decomposição da serapilheira e a liberação de nutrientes foi quantificada por meio de litter bags. Para a avaliação da atividade microbiana procedeu-se à quantificação do carbono mineralizável por meio da evolução de $\mathrm{CO}_{2}$. Os resultados mostraram que as coberturas florestais se comportaram de forma diferenciada quanto à decomposição e atividade microbiana, destacando-se a Sapindus saponaria, que apresentou maior velocidade de decomposição de serapilheira. A cobertura de Sapindus saponaria apresentou quantidades acumuladas de $\mathrm{CO}_{2}$ superiores às demais. A cobertura de Acacia mangium apresentou os menores valores de $\mathrm{CO}_{2}$ acumulado.
\end{abstract}

Palavras-chave: ecossistema florestal, ciclagem de nutrientes, microrganismo.

\section{Decomposition and Microbial Activity of Forest Floor Litter in the South of Espírito Santo}

\begin{abstract}
The decomposition process of litter should be widely studied, as it is a key factor for nutrient maintenance and cycling in ecosystems. This study aimed to evaluate the decomposition, release of nutrients, and microbial activity of the forest floor litter of secondary forest, Acacia mangium, Sapindus saponaria, and Hevea brasiliensis in the south of Espirito Santo. The litter decomposition and nutrients release was measured by means of litter bags added to each forest cover. For the assessment of microbial activity, we quantified mineralizable carbon by $\mathrm{CO}_{2}$ evolution. The results showed that the forest covers behaved differently in terms of decomposition and microbial activity; particular emphasis is given to Sapindus saponaria, which presented a faster decomposition of litter. The cover of Sapindus saponaria also presented higher amounts of accumulated $\mathrm{CO}_{2}$ compared to other forest covers; whereas the cover of Acacia mangium presented the lowest values of accumulated $\mathrm{CO}_{2}$.
\end{abstract}

Keywords: forest ecosystem, nutrient cycling, microorganism. 


\section{INTRODUÇÃO}

O processo de deposição de serapilheira, incluindo as taxas de queda do material decíduo e seu processo de decomposição, deve ser amplamente estudado e conhecido, por se tratar de um fator-chave na manutenção e ciclagem de nutrientes nos ecossistemas, principalmente em regiões tropicais onde, de maneira geral, os solos apresentam baixa fertilidade natural (Santana \& Souto, 2011). Além disso, estudos relacionados à produção e acúmulo de serapilheira fornecem subsídios para melhor entendimento da dinâmica de nutrientes (Godinho et al., 2014). A decomposição da serapilheira é controlada, principalmente, por fatores como a temperatura e precipitação (Dickinson, 1974; Jensen, 1974), condições físicas e químicas do ambiente, qualidade orgânica e nutricional do material que é aportado, além da composição da comunidade detritívora e sua afinidade com o substrato (Cianciaruso et al., 2006). Estudos realizados em florestas tropicais evidenciam que as taxas de decomposição são muito afetadas pelas variações sazonais, formando padrões distintos nas estações chuvosa e seca (Cornu et al., 1997). De acordo com Barros \& Comerford (2002), a comparação entre o processo de ciclagem de nutrientes em floresta plantada com o da floresta natural permite avaliar possíveis alterações que ocorrem em decorrência de técnicas de manejo aplicadas e interferências sobre a sustentabilidade das plantações. Ainda segundo os mesmos autores, em solos de baixa fertilidade, o acúmulo e a decomposição da serapilheira podem servir como indicadores de diferenças entre ecossistemas, principalmente no que se refere à disponibilidade de nutrientes. A concentração $\mathrm{e}$ o conteúdo de nutrientes na serapilheira podem variar de acordo com alguns fatores como tipo de solo, da vegetação, densidade populacional, habilidade de a espécie absorver, utilizar e redistribuir os nutrientes e de acordo com a idade das árvores (Cunha et al., 2013). De modo geral, regiões que apresentam elevada precipitação pluviométrica apresentam maior produção de serapilheira se comparadas a regiões mais secas (Inknotte et al., 2015).

A taxa de decomposição da serapilheira $(\mathrm{k})$ indiretamente representa a velocidade com que os nutrientes contidos nela tornam-se disponíveis, sendo considerada alta quando os valores de $\mathrm{k}$ são acima de 1,0 (Waring \& Schlesinger, 1985). De acordo com Correia \& Andrade (1999), o valor ké um dos principais índices utilizados para estimar a deposição de serapilheira em coberturas florestais. Cianciaruso et al. (2006) discutem que as serapilheiras possuem composições distintas e, consequentemente, velocidade de decomposição diferentes, sendo a velocidade global de decomposição dependente da proporção dos diferentes componentes presentes. Elevados teores de alguns componentes químicos como lignina, polifenóis e celulose podem retardar a taxa de decomposição da serapilheira, resultando em menores níveis de nutrientes no solo (Swift et al.,1979).

Os objetivos deste trabalho foram: avaliar a decomposição da serapilheira, a liberação de nutrientes e quantificar e avaliar a dinâmica do carbono mineralizável em quatro coberturas florestais (Acacia mangium Willd., Sapindus saponaria L., Hevea brasiliensis e floresta secundária) na região sul do Estado do Espírito Santo.

\section{MATERIAL E MÉTODOS}

\subsection{Caracterização do local de estudo}

O estudo foi conduzido na Fazenda Experimental do Incaper, localizada a $30 \mathrm{~km}$ da cidade de Cachoeiro de Itapemirim, ES. As coordenadas da área são $20^{\circ} 45^{\prime} 17.31^{\prime \prime}$ S e $41^{\circ} 17^{\prime} 8.86^{\prime \prime} \mathrm{W}$, com altitude de $100 \mathrm{~m}$.

O clima da região é do tipo Cwa, apresentando chuva mal distribuída ao longo do ano, com verão chuvoso e inverno seco, de acordo com a classificação de Köpen. Os períodos de maior índice pluviométrico iniciam-se aproximadamente na segunda quinzena de outubro e se prolongam até a primeira quinzena de março (Figura 1), sendo os meses de novembro e dezembro os mais chuvosos (Oliveira, 2007).

Para o estudo foram selecionadas quatro áreas $(20 \times 30 \mathrm{~m})$ com as seguintes coberturas florestais: floresta secundária, Acacia mangium Willd., Sapindus saponaria L. e Hevea brasiliensis. As áreas com Acacia mangium e Sapindus saponaria faziam parte do Projeto de Recuperação de Áreas Degradadas com Espécies Florestais, desenvolvido pelo Incaper e implantado no ano de 1994. Anteriormente, a área era ocupada por pasto. Os dois plantios possuem uma área de $20 \times 30 \mathrm{~m}$ e espaçamento de 2,0 × 2,5 m. A área com Hevea brasiliensis possui aproximadamente 3 ha e foi implantada em dezembro de 2003, sobre pastagem cultivada anteriormente, sendo utilizado um espaçamento de 8,0 x 2,5 m e o clone Fx 386. Na área sob floresta secundária ocorre dominância das espécies arbóreas 
das famílias Fabaceae, Sapotaceae, Euphorbiaceae, Myrtaceae e Meliaceae (Oliveira, 2007).

A área experimental encontra-se sobre Latossolo Vermelho Amarelo distrófico de textura argilosa (EMBRAPA, 1999). Os atributos químicos desse solo são apresentados na Tabela 1.

\subsection{Decomposição de serapilheira - taxa de decomposição $(k)$ e liberação de nutrientes}

Para a avaliação da taxa de decomposição do material aportado ao solo, no mês de novembro de 2010 foram coletadas folhas senescentes pertencentes a cada área em estudo. Após coletado, o material foi seco em estufa a $65^{\circ} \mathrm{C}$, pesado (10 gramas) e colocado, separadamente, em litter bags (malha de $2 \mathrm{~mm}$ e dimensões de $20 \times 20 \mathrm{~cm}$ ). Seis litter bags foram colocados aleatoriamente sobre a superfície do solo em cada cobertura florestal. Para cada cobertura florestal foram realizadas três repetições, totalizando 18 litter bags por cobertura florestal.

Os litter bags foram coletados 30, 75, 105, 135, 165 e 225 dias após o lançamento nas áreas. Após cada coleta, as amostras de serapilheira foram levadas para o Laboratório de Solos do CCA-UFES, onde foram secas

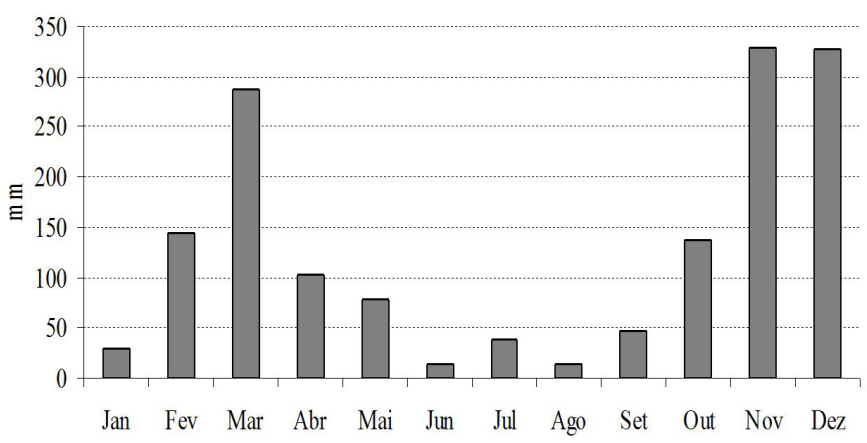

Figura 1. Precipitação pluviométrica $(\mathrm{mm})$ no ano de 2010 para a localização da Fazenda Experimental Bananal do Norte, pertencente ao Incaper, no sul do Estado do Espírito Santo. Fonte: INCAPER (2010).

Figure 1. Rainfall (mm) in 2010 for the location of the Experimental Farm Bananal do Norte, belonging to Incaper, in the southern state of Espirito Santo. Source: INCAPER (2010).

Tabela 1. Atributos químicos do solo coletado nas profundidades $0-10 \mathrm{~cm}$ e $10-20 \mathrm{~cm}$ para cada cobertura florestal estudada (floresta secundária, Acacia mangium, Sapindus saponaria e Hevea brasiliensis) na região sul do Espírito Santo. Table 1. Soil chemical properties collected from depths of 0 to 10 and 10 to $20 \mathrm{~cm}$ for each studied forest cover (secondary forest, Acacia mangium, Sapindus saponaria and Hevea brasiliensis) in southern Espirito Santo.

\begin{tabular}{|c|c|c|c|c|c|c|c|c|}
\hline \multirow[t]{3}{*}{ Atributos } & \multicolumn{2}{|c|}{ Floresta secundária } & \multicolumn{2}{|c|}{ Sapindus saponaria } & \multicolumn{2}{|c|}{ Acácia mangium } & \multicolumn{2}{|c|}{ Hevea brasiliensis } \\
\hline & & - & 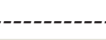 & fundida & (cm) & -----. & ------ & --- \\
\hline & $0-10$ & $10-20$ & 0-10 & $10-20$ & $0-10$ & $10-20$ & 0-10 & $10-20$ \\
\hline $\mathrm{pH}\left(\mathrm{H}_{2} \mathrm{O}\right)^{1}$ & 6,2 & 5,9 & 6,0 & 5,9 & 5,9 & 6,0 & 5,0 & 5,0 \\
\hline $\mathrm{P}\left(\mathrm{mg} \mathrm{dm}^{-3}\right)^{2}$ & 18,8 & 9,0 & 12,0 & 6,4 & 12,2 & 11,5 & 13,9 & 7,5 \\
\hline $\mathrm{K}\left(\mathrm{mg} \mathrm{dm}^{-3}\right)^{2}$ & 165 & 109 & 47 & 33 & 97,0 & 67 & 118,0 & 33 \\
\hline $\mathrm{Na}\left(\mathrm{cmol}_{\mathrm{c}} \mathrm{dm}^{-3}\right)^{2}$ & 0,18 & 0,12 & 0,15 & 0,14 & 0,16 & 0,16 & 0,15 & 0,15 \\
\hline $\mathrm{Ca}^{+2}\left(\mathrm{cmol}_{\mathrm{c}} \mathrm{dm}^{-3}\right)^{3}$ & 6,55 & 3,37 & 5,01 & 3,37 & 6,38 & 4,81 & 1,88 & 1,79 \\
\hline $\mathrm{Mg}^{+2}\left(\mathrm{cmol}_{\mathrm{c}} \mathrm{dm}^{-3}\right)^{3}$ & 3,54 & 2,60 & 2,78 & 2,22 & 2,84 & 2,50 & 2,63 & 1,77 \\
\hline $\mathrm{Al}^{+3}\left(\mathrm{cmol}_{\mathrm{c}} \mathrm{dm}^{-3}\right)^{3}$ & 0,27 & 0,15 & 0,25 & 0,19 & 1,10 & 0,05 & 0,62 & 0,36 \\
\hline $\mathrm{H}+\mathrm{Al}\left(\mathrm{cmol}_{\mathrm{c}} \mathrm{dm}^{-3}\right)^{4}$ & 3,19 & 2,70 & 2,86 & 3,14 & 5,06 & 3,47 & 4,13 & 3,41 \\
\hline C.O. $\left(\text { dag kg-1 }^{-1}\right)^{5}$ & 2,14 & 1,34 & 1,72 & 1,41 & 2,43 & 1,65 & 1,39 & 1,16 \\
\hline $\mathrm{CTC}^{6}$ & 13,60 & 9,07 & 10,92 & 8,96 & 14,69 & 11,12 & 9,15 & 7,21 \\
\hline $\mathrm{SB}\left(\mathrm{cmol}_{\mathrm{c}} \mathrm{dm}^{-3}\right)$ & 10,41 & 6,37 & 8,06 & 5,82 & 9,63 & 7,65 & 5,02 & 3,80 \\
\hline V (\%) & 76,55 & 70,24 & 73,81 & 64,95 & 65,55 & 68,79 & 54,84 & 52,70 \\
\hline
\end{tabular}

${ }^{1}$ Relação solo-água 1:2,5; ${ }^{2}$ Extrator Mehlich- $1 ;{ }^{3}$ Extrator $\mathrm{KCl} 1 \mathrm{~mol} \mathrm{~L}{ }^{-1} ;{ }^{4}$ Extrator $\mathrm{Ca}(\mathrm{Oac}){ }_{2} 0,5 \mathrm{~mol} / \mathrm{L} \mathrm{pH} \mathrm{7,0;}{ }^{5} \mathrm{C} . \mathrm{O}$. = carbono orgânico, determinado por oxidação via úmida, com dicromato de potássio em meio sulfúrico (Yeomans \& Bremner, 1988); ${ }^{6} \mathrm{CTC}$ - capacidade de troca catiônica calculada pelo somatório dos cátions trocáveis: $\mathrm{Ca}^{+2}, \mathrm{Mg}^{+2}, \mathrm{~K}, \mathrm{Na}^{+}$e $\mathrm{H}+\mathrm{Al} ; \mathrm{SB}=$ soma de bases; $\mathrm{V} \%=$ saturação de bases. 
em estufa a $65^{\circ} \mathrm{C}$ e pesadas posteriormente, obtendo-se o peso da matéria seca (MS). Em seguida, a MS foi moída e obteve-se uma amostra, que foi submetida à digestão nitroperclórica (Tedesco et al., 1995). Na alíquota obtida da digestão foram determinados os teores de Ca e Mg, através do uso de espectrofotômetro de absorção atômica, de K, pelo fotômetro de chama, e de P, pelo espectrofotômetro de absorção visível, conforme Embrapa (1997). Os resultados foram analisados pelo Teste t de Student a 5\% de probabilidade.

A partir desses dados foram determinados os percentuais de matéria seca (MS) remanescentes em relação às quantidades adicionadas inicialmente. Foram ajustados modelos para avaliar a taxa de decomposição, de acordo com a seguinte equação proposta por Thomas \& Asakawa (1993): XRt $=\mathrm{a} \exp ^{(-\mathrm{kt})}$, em que XRt é o peso seco ou nutriente restante a um tempo " $t$ " e uma constante $k$ de decomposição. O parâmetro "a" corresponde ao ponto de máximo da função e representa a quantidade inicial de MS ou de nutrientes adicionada aos litter bags. $\mathrm{O}_{1 / 2}$ foi calculado através do logaritmo neperiano dividido pelo valor da taxa $\mathrm{k}$.

\subsection{Determinação do carbono mineralizável}

A quantificação do carbono mineralizável foi realizada por meio da evolução de $\mathrm{CO}_{2}$ produzido pela atividade microbiana, capturado em solução de $\mathrm{NaOH}$ 0,5 mol L-1, segundo método proposto por Anderson (1982).

Para esse experimento utilizou-se o delineamento experimental inteiramente casualizado, com arranjo fatorial $4 \times 2$ (quatro coberturas florestais e duas profundidades). Recipientes plásticos com tampa de rosca $(250 \mathrm{ml})$, contendo 50 gramas de material de solo seco e passado em peneira de $2 \mathrm{~mm}$ e 5 gramas de matéria seca da serapilheira de cada cobertura florestal moída e peneirada em peneira de $2 \mathrm{~mm}$ foram umedecidos a $70 \%$ da capacidade de campo, constituindo uma unidade experimental. O estudo foi realizado com solo coletado nas profundidades de 0-10 cm e 10-20 cm.

Cada recipiente recebeu $10 \mathrm{~mL}$ da solução de $\mathrm{NaOH}$ $0,5 \mathrm{~mol} \mathrm{~L}^{-1}$ acondicionada em copos plásticos de $50 \mathrm{~mL}$. Esses recipientes foram hermeticamente fechados para a captura do $\mathrm{CO}_{2}$ liberado, sendo abertos somente por ocasião da troca da solução de $\mathrm{NaOH}$. As trocas das soluções de $\mathrm{NaOH}$ foram realizadas em intervalos de $5,15,25,35$ e 45 dias.

\subsection{Análise estatística}

Os resultados foram submetidos à análise de variância, sendo que os efeitos dentro dos fatores qualitativos (espécies) foram desdobrados em contrastes e a curva de evolução de $\mathrm{CO}_{2}$ (produção acumulada de $\mathrm{CO}_{2}$ ) com o tempo de incubação foram ajustados a equações logísticas $\mathrm{Y}=\mathrm{a} / 1+\mathrm{e}^{-(\mathrm{b}+\mathrm{cx})}$.

A curva de evolução de $\mathrm{CO}_{2}$ (produção acumulada de $\mathrm{CO}_{2}$ ) com o tempo de incubação foram ajustados na equação logística $\mathrm{Y}=\mathrm{a} / 1+\mathrm{e}^{-(\mathrm{b}+\mathrm{cx})}$, em que o coeficiente $a$ é a saturação da curva associada à máxima evolução de $\mathrm{CO}_{2}$ e indica a quantidade de $\mathrm{C}$ mais facilmente mineralizável; $b$ indica o deslocamento da curva horizontalmente, e quanto maior o seu valor, maior é o tempo para atingir a saturação; o coeficiente $c$ está relacionado com a taxa de crescimento da função, diretamente associado à labilidade do $\mathrm{C}$ na matéria orgânica. Por intermédio desses coeficientes foi possível estimar o tempo necessário para se atingir a metade da produção máxima de $\mathrm{CO}_{2}\left(\mathrm{t}_{1 / 2}\right)$; seu valor permite que se faça uma inferência sobre a velocidade de mineralização da matéria orgânica do solo e, consequentemente, sobre sua labilidade (Passos et al., 2007; Andrade et al., 2013).

\section{RESULTADOS E DISCUSSÃO}

\subsection{Decomposição de serapilheira - taxa de decomposição (k) e matéria seca remanescente}

É possível observar maior decomposição inicial da serapilheira nos primeiros dias do estudo (Figura 2). Resultados semelhantes também foram verificados por Silva et al. (2013) em um fragmento de Mata Atlântica no sul do Espírito Santo. Para Lupwayi et al. (2004), esse é o período em que ocorre a maior perda de compostos solúveis mais lábeis e de fácil decomposição. O processo de decomposição segue um modelo exponencial, indicando que o processo não é constante ao longo do ano, sendo maior nos meses iniciais. Silva et al. (2014) observaram redução de 17\% de biomassa vegetal nos primeiros meses do experimento, assim como observado por outros autores nos primeiros meses de avaliação (Fernandes et al., 2006; Pegado et al., 2008; Cunha et al., 2013).

Após o período de 30 dias, 14,6\% da MS da floresta secundária dentro dos litter bags foi decomposta, 


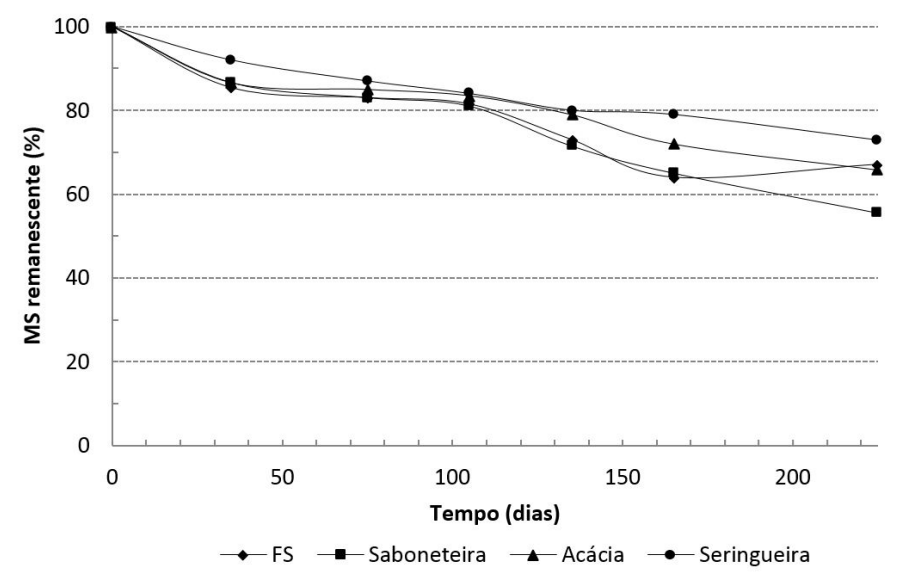

Figura 2. Curvas de decomposição da serapilheira para cada cobertura florestal estudada (floresta secundária, Acacia mangium, Sapindus saponaria e Hevea brasiliensis) na região sul do Espírito Santo.

Figure 2. Litter decomposition curves for each forest cover studied (Secondary forest, Acacia mangium, Sapindus saponaria and Hevea brasiliensis) in the south of Espírito Santo.

enquanto que para as coberturas Sapindus saponaria (saboneteira), Acacia mangium (acácia mangium) e Hevea brasiliensis (seringueira) os valores foram de $13,4 \%, 13,4 \%$ e $8,0 \%$, respectivamente (Figura 2).

A maior decomposição inicial da serapilheira da floresta secundária pode ser explicada pelo fato de que a diversidade de espécies vegetais presentes num determinado local influencia o processo de decomposição na medida em que afeta a qualidade do substrato (folhas e raízes, principalmente), o ambiente de decomposição (microclima) e, assim, a atividade da comunidade decompositora.

Entre 30 e 75 dias de avaliação da decomposição da serapilheira observou-se baixa redução de MS com destaque para a cobertura seringueira, o que pode estar relacionado à permanência dos compostos mais recalcitrantes ao ataque microbiano, paralelamente à rápida decomposição inicial da fração mais facilmente decomposta (Silva et al., 2014). Carvalho et al. (2008) descrevem que a susceptibilidade dos resíduos à deterioração está relacionada a fatores associados à sua composição química, como teor de celulose, de hemicelulose, de lignina e de polifenóis. No processo de decomposição dos resíduos, ocorre primeiramente a biodegradação rápida da maioria dos compostos hidrossolúveis e polissacarídeos, redução lenta de hidrossolúveis fenólicos e hemiceluloses e aumento relativo do conteúdo de ligninas e proteínas (Correia \& Andrade, 1999).
Gonçalves et al. (2011) relatam que variações sazonais também podem promover alterações nas taxas de decomposição do material, o que justifica os resultados observados no presente estudo. O período de menor ocorrência de chuvas influenciou negativamente a atividade microbiológica do solo, reduzindo a taxa de decomposição do material vegetal.

A perda de MS no final do experimento, aos 225 dias, foi de 34,18\% na floresta secundária, 44,49\% na saboneteira, $32,92 \%$ na Acacia mangium e 27,08\% na seringueira.

Verificou-se menor constante $\mathrm{k}$ para seringueira $(0,0013)$ (Tabela 2), não distante da taxa k da Acacia mangium $(0,0016)$. Não houve grande diferença também entre a taxa $\mathrm{k}$ para floresta secundária $(0,0017)$ e saboneteira $(0,0024)$ indicando, portanto, que a serapilheira da saboneteira e floresta secundária apresentam velocidade de decomposição semelhante.

Cunha et al. (2013), avaliando a decomposição de espécies florestais, encontrou valor de $\mathrm{k}$ igual a 0,0013 para a Acacia mangium e de 0,0026 para floresta secundária. No mesmo estudo, os valores de $\mathrm{t}_{1 / 2}$ obtidos foram de 533 dias e 266 dias para Acacia mangium e floresta secundária, respectivamente. Ainda segundo os mesmo autores, o elevado tempo de meia vida verificado para áreas de acácia, associado ao reduzido valor da taxa de decomposição (k) explicam o acúmulo da fração foliar nessas áreas, assim como observado no presente estudo. Silva et al. (2014), em 
estudo semelhante sob áreas de vegetação nativa, observaram valores de $\mathrm{k}(0,0019$ a 0,0023) similares aos apresentados neste trabalho.

A taxa $\mathrm{k}$ para a seringueira diferiu das coberturas floresta secundária e saboneteira (Tabela 2), apresentando menor velocidade de decomposição e, portanto, demandando maior de tempo para que os nutrientes sejam disponibilizados para o solo.

Fernandes et al. (2006) verificaram que áreas com presença de leguminosas arbóreas (Acacia mangium e saboneteira) apresentam maior velocidade de decomposição, padrão esse que não foi verificado para a acácia no presente estudo. Esse fato pode ser atribuído à baixa velocidade de decomposição dos filódios (folhas modificadas) presentes na maior parte da serapilheira produzida por essa espécie, assim como observaram Balieiro et al. (2004), que, utilizando o mesmo método,

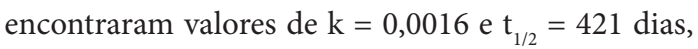
valores tão elevados quanto os encontrados no presente trabalho $\left(\mathrm{k}=0,0016\right.$ e $_{1 / 2}=497,64$ dias $)$.

Valente et al. (2005), avaliando diferentes plantios florestais, constataram que os plantios que apresentavam Acacia auriculiformes e Acacia mangium em sua composição mostraram maior dificuldade de decomposição do material decíduo do que o plantio sem essas espécies.

\subsection{Decomposição de serapilheira - liberação de nutrientes}

Em solos de baixa fertilidade, o acúmulo e a decomposição da serapilheira podem servir de indicadores de diferenças entre sistemas puros e mistos, em especial no que tange à disponibilidade de nutrientes para as plantas (Gama-Rodrigues et al.,
2003). Para o Ca observou-se um comportamento similar na liberação desse nutriente para todas as coberturas florestais (Tabela 3). Em geral, a liberação de Ca diminuiu com o decorrer dos dias, ou seja, com a decomposição da matéria seca, observado pela redução dos valores ao longo do tempo. Esse fato pode ser justificado por esse nutriente ser um componente estrutural das células do tecido vegetal, tendendo assim a ser um dos últimos nutrientes a ser liberado para o solo através da decomposição da serapilheira (Godinho et al., 2014). Diante disso, quanto maior a decomposição da serapilheira, principalmente de folhas, maior a liberação de Ca (Cobo et al., 2002). Silva et al. (2013) discutem que o Ca, por ser um elemento de baixa mobilidade na planta, retorna ao solo em grande quantidade com a queda dos galhos, folhas e frutos.

Para o Mg, a cobertura que apresentou liberação contínua desse nutriente foi a seringueira (Tabela 3). De acordo com Vieira et al. (2014), a liberação de magnésio não acompanha a perda de massa da serapilheira, evidenciando o comportamento instável desse nutriente durante a decomposição do material vegetal. Ainda segundo Colle \& Rapp (1980), o conteúdo de magnésio é variável entre as fitofisionomias florestais, não obedecendo, portanto, a um padrão estabelecido.

Em relação ao nutriente $\mathrm{K}$, observa-se para todas as coberturas o mesmo comportamento (Tabela 3), no qual esse nutriente apresentou maior liberação nos primeiros dias e, em contrapartida, liberação mais lenta ao final do período de avaliação. Uma possível explicação para esse comportamento pode estar relacionada ao fato de o período de menor liberação do conteúdo de $\mathrm{K}$ coincidir com as menores taxas de precipitação pluviométrica, uma vez que o K não está

Tabela 2. Estimativa dos parâmetros $(\mathrm{a}, \mathrm{k})$ da equação de decomposição e do tempo de meia-vida $\left(\mathrm{t}_{1 / 2}\right)$ para cada cobertura florestal estudada (floresta secundária, Acacia mangium, Sapindus saponaria e Hevea brasiliensis) na região sul do Espírito Santo.

Table 2. Estimation of the parameters $(\mathrm{a}, \mathrm{k})$ of the equation and the decomposition half-life ( $\mathrm{t} 1 / 2)$ for each forest cover studied (Secondary forest, Acacia mangium, Sapindus saponaria and Hevea brasiliensis) in the south of Espírito Santo.

\begin{tabular}{lllll}
\multicolumn{1}{c}{ Cobertura florestal } & $\boldsymbol{a}(\mathbf{g})$ & $\mathbf{k}\left(\mathbf{d i a}^{-1}\right)$ & $\mathbf{R}^{2}$ & $\mathbf{t}_{\mathbf{1 / 2}}(\mathbf{d i a s})$ \\
\hline & & $\mathbf{M S}$ & & $394,36 \mathrm{ab}$ \\
\hline Floresta secundária & 9,4352 & $0,0017 \mathrm{a}$ & 0,85 & $292,75 \mathrm{~b}$ \\
Sapindus saponaria & 9,8529 & $0,0024 \mathrm{a}$ & 0,95 & $497,64 \mathrm{ab}$ \\
Acacia mangium & 9,6349 & $0,0016 \mathrm{a} \mathrm{b}$ & 0,88 & $529,06 \mathrm{a}$ \\
\hline Hevea brasiliensis & 9,6830 & $0,0013 \mathrm{~b}$ & 0,89 & \\
\hline
\end{tabular}

Médias seguidas da mesma letra, na coluna, não diferem estatisticamente entre si pelo teste t a $5 \%$ de probabilidade. 
Tabela 3. Conteúdo de nutrientes (mg) liberados durante a decomposição da serapilheira para cada cobertura florestal estudada (floresta secundária, Acácia mangium, Sapindus saponaria e Hevea brasiliensis) na região sul do Espírito Santo.

Table 3. Nutrient content (mg) remaining after each period of decomposition of litter for each forest cover studied (Secondary forest, Acacia mangium, Sapindus saponaria and Hevea brasiliensis) in the south of Espírito Santo.

\begin{tabular}{|c|c|c|c|c|c|c|c|}
\hline Cobert. florestal & $\mathbf{0}$ & 30 & 75 & 105 & 135 & 165 & 225 \\
\hline & \multirow{2}{*}{\multicolumn{7}{|c|}{$\mathbf{P}$}} \\
\hline Floresta secundária & & 50 & 77 & & 40 & 42 & 35 \\
\hline Sanind congria & $1,0 \mathrm{~d}$ & $3,0 \mathrm{~d}$ & $4,1 \mathrm{a}$ & 4,9 d & $4,0 \mathrm{~d}$ & $4,5 \mathrm{a}$ & $5,5 \mathrm{~d}$ \\
\hline Sapindus saponaria & $5,4 \mathrm{a}$ & $4,0 \mathrm{a}$ & $4,5 \mathrm{a}$ & $4,9 \mathrm{a}$ & $4,0 \mathrm{a}$ & $4,2 \mathrm{a}$ & $3,7 \mathrm{a}$ \\
\hline Acacia mangium & $3,8 \mathrm{a}$ & $2,4 \mathrm{~b}$ & $2,9 \mathrm{~b}$ & $2,7 \mathrm{~b}$ & $2,5 \mathrm{~b}$ & $3,0 \mathrm{a}$ & $1,5 \mathrm{~b}$ \\
\hline \multirow[t]{2}{*}{ Hevea brasiliensis } & $6,2 \mathrm{a}$ & $4,2 \mathrm{a}$ & $4,5 \mathrm{a}$ & $3,9 \mathrm{ab}$ & $3,7 \mathrm{ab}$ & $3,8 \mathrm{a}$ & $1,8 \mathrm{~b}$ \\
\hline & \multicolumn{7}{|c|}{$\mathrm{Ca}$} \\
\hline Floresta secundária & $6,4 \mathrm{a}$ & $5,2 \mathrm{a}$ & $5,1 \mathrm{a}$ & $5,2 \mathrm{a}$ & $1,8 \mathrm{~b}$ & $4,4 \mathrm{a}$ & $3,9 \mathrm{a}$ \\
\hline Sapindus saponaria & $4,9 \mathrm{ab}$ & $5,6 \mathrm{a}$ & $5,0 \mathrm{a}$ & $6,2 \mathrm{a}$ & $4,6 \mathrm{a}$ & $4,2 \mathrm{a}$ & $3,5 \mathrm{a}$ \\
\hline Acacia mangium & $2,6 \mathrm{~b}$ & $4,8 \mathrm{a}$ & $3,3 \mathrm{~b}$ & $4,0 \mathrm{a}$ & $4,1 \mathrm{a}$ & $3,5 \mathrm{a}$ & $2,7 \mathrm{~b}$ \\
\hline \multirow[t]{2}{*}{ Hevea brasiliensis } & $6,2 \mathrm{a}$ & $5,3 \mathrm{a}$ & $5,0 \mathrm{a}$ & $4,3 \mathrm{a}$ & $3,5 \mathrm{ab}$ & $4,5 \mathrm{a}$ & $2,9 \mathrm{~b}$ \\
\hline & \multicolumn{7}{|c|}{$\mathrm{Mg}$} \\
\hline Floresta secundária & $8,4 \mathrm{a}$ & $6,7 \mathrm{a}$ & $6,5 \mathrm{a}$ & $6,5 \mathrm{a}$ & $4,3 \mathrm{a}$ & $5,2 \mathrm{a}$ & $6,0 \mathrm{a}$ \\
\hline Sapindus saponaria & $7,2 \mathrm{a}$ & $6,2 \mathrm{a}$ & $5,2 \mathrm{a}$ & $6,1 \mathrm{a}$ & $5,1 \mathrm{a}$ & $4,5 \mathrm{a}$ & $5,2 \mathrm{a}$ \\
\hline Acacia mangium & $4,9 \mathrm{a}$ & $6,3 \mathrm{a}$ & $4,6 \mathrm{a}$ & $5,3 \mathrm{a}$ & $5,1 \mathrm{a}$ & $4,3 \mathrm{a}$ & $3,8 \mathrm{a}$ \\
\hline \multirow[t]{2}{*}{ Hevea brasiliensis } & $6,7 \mathrm{a}$ & $8,5 \mathrm{a}$ & $7,2 \mathrm{a}$ & $6,6 \mathrm{a}$ & $6,3 \mathrm{a}$ & $5,4 \mathrm{a}$ & $4,6 \mathrm{a}$ \\
\hline & \multicolumn{7}{|c|}{$\mathbf{K}$} \\
\hline Floresta secundária & $21,3 \mathrm{a}$ & $15,5 \mathrm{a}$ & $18,0 \mathrm{a}$ & $15,3 \mathrm{a}$ & $12,9 \mathrm{ab}$ & $15,4 \mathrm{a}$ & $29,9 \mathrm{a}$ \\
\hline Sapindus saponaria & $13,3 \mathrm{~b}$ & $13,3 \mathrm{~b}$ & $12,8 \mathrm{~b}$ & $12,3 \mathrm{~b}$ & $13,3 \mathrm{a}$ & $9,1 \mathrm{~b}$ & $29,6 \mathrm{a}$ \\
\hline Acacia mangium & $15,8 \mathrm{~b}$ & $13,4 \mathrm{~b}$ & $13,2 \mathrm{~b}$ & $10,6 \mathrm{~b}$ & $13,0 \mathrm{a}$ & $7,3 \mathrm{~b}$ & $32,1 \mathrm{a}$ \\
\hline Hevea brasiliensis & $14,3 \mathrm{~b}$ & $10,4 \mathrm{~b}$ & $10,8 \mathrm{~b}$ & $7,8 \mathrm{~b}$ & $6,8 \mathrm{~b}$ & $7,5 \mathrm{~b}$ & $28,8 \mathrm{a}$ \\
\hline
\end{tabular}

Valores seguidos da mesma letra, na coluna, não diferem estatisticamente entre si pelo teste t a $5 \%$ de probabilidade.

associado a nenhum componente estrutural do tecido vegetal, sendo facilmente mineralizável (Taiz \& Zeiger, 2009). De acordo com Selle (2007), as maiores taxas de transferência de $\mathrm{K}$ ocorreram com a chegada das primeiras chuvas.

Resultados semelhantes foram encontrados por Silva et al. (2013), que observaram maior liberação de $\mathrm{K}$ nos primeiros 90 dias do estudo em fragmento de Floresta Atlântica no sul do Espírito Santo

Para os valores de P em todas as coberturas foi possível observar maior liberação nos primeiros 30 dias, ocorrendo em seguida diminuição na liberação desse elemento (Tabela 3). Gama-Rodrigues et al. (2003) observaram menor liberação de fósforo em uma capoeira no sudeste da Bahia em relação a uma floresta natural, sendo o processo de liberação e decomposição de nutrientes regulados pela diversidade do substrato e do microambiente.

É possível observar que a Acacia mangium apresentou menor taxa de liberação de nutrientes e, consequentemente, menor taxa decomposição da serapilheira, fato confirmado por a espécie apresentar um dos menores valores de $\mathrm{k}$. Essa característica pode ser visualizada na Tabela 2 , em que ela apresenta o segundo menor valor para a taxa de decomposição. Esse fato pode ser justificado pelo alto teor de lignina que a acácia apresenta, como relatado por Bini et al. (2013). Para os autores, os resíduos de acácia são menos susceptíveis à decomposição rápida devido ao maior teor de lignina, que é um material mais fibroso, com altos níveis de nitrogênio. Moreira \& Siqueira (2006) discutem que elevados teores de lignina e compostos aromáticos promovem aumento da resistência do material à decomposição por organismos do solo, ao passo que elevados teores de carboidratos solúveis ou celulose diminuem a resistência, favorecendo o aumento da taxa de decomposição.

\subsection{Carbono mineralizável}

Observou-se variação na quantidade acumulada de $\mathrm{CO}_{2}$ durante o período de incubação (45 dias), constatando-se diferenças entre as coberturas florestais 
(Tabela 4). Valores mais elevados na produção de $\mathrm{CO}_{2}$ implicam em maior atividade biológica, que está diretamente relacionada com a disponibilidade de carbono do solo e da biomassa microbiana (Mercante et al., 2004). Souto (2006) descrevem que a atividade biológica na mineralização dos resíduos orgânicos é refletida na respiração edáfica.

Para as duas profundidades do solo estudadas observou-se que as quantidades acumuladas de $\mathrm{CO}_{2}$ foram superiores para a cobertura de saboneteira.

A Acacia mangium foi a cobertura florestal que apresentou menor valor de produção acumulada de $\mathrm{CO}_{2}$ total $\left(3,67 \mathrm{~g} \mathrm{~kg}^{-1}\right.$ e $\left.3,59 \mathrm{~g} \mathrm{~kg}^{-1}\right)$ para as profundidade 0-10 cm e 10-20 cm, respectivamente, indicando que essa espécie pode apresentar serapilheira mais resistente à ação de microrganismos, portanto, menor labilidade. É possível associar esses valores com a baixa taxa $\mathrm{k}$ dessa cobertura florestal.

Esperava-se encontrar maiores valores de produção acumulada de $\mathrm{CO}_{2}$ para a cobertura floresta secundária. Porém esses resultados podem estar relacionados ao fato de a serapilheira das coberturas florestais terem sido moídas e peneiradas, proporcionando a mesma superfície específica para todas as serapilheiras. Além disso, Bini et al. (2013) observaram menor taxa de decomposição do material decíduo em plantios contendo diferentes espécies, atribuindo a dificuldade de decomposição à mistura dos materiais vegetais. Smith \& Bradford (2003) ressaltam que a mistura de materiais pode ter efeito negativo sobre a decomposição dos resíduos vegetais.

Os valores dos contrastes para a produção acumulada de $\mathrm{CO}_{2}$ para as coberturas florestais podem ser visualizados na Tabela 5 .

No contraste $\mathrm{Cl}$ (Tabela 5), para as duas profundidades do solo avaliadas não foram observadas diferenças significativas entre as quantidades acumuladas de $\mathrm{CO}_{2}$. Pelo contraste C2 (Tabela 5), observou-se diferença significativa entre as quantidades acumuladas de $\mathrm{CO}_{2}$, sendo que maior produção acumulada de $\mathrm{CO}_{2}$ foi observada para cobertura de saboneteira. A mesma espécie obteve maior valor para a taxa de decomposição, como se observa na Figura 2 e na Tabela 2.

Gama-Rodrigues et al. (2008) trabalhando com atividade microbiana em área com Acacia auriculiformis, encontraram valores de $0,031 \mathrm{~g} / \mathrm{kg}$ para produção acumulada de $\mathrm{CO}_{2}$ em um período de 7 dias de incubação. Porém esses autores trabalharam apenas com solo, não incorporando a serapilheira. Ainda segundos esses mesmos autores, foram encontrados valores de $0,058 \mathrm{~g} / \mathrm{kg}$ e $0,064 \mathrm{~g} / \mathrm{kg}$ para pastagem e capoeira, respectivamente. Geralmente, os valores para a atividade microbiana são menores quando se trabalha apenas com o solo. Isso pode ser explicado por a presença da matéria orgânica da serapilheira favorecer o aumento a biomassa e a atividade da microbiota do solo. Loss et al. (2013) discutem que a adição de resíduos orgânicos e/ou maior disponibilização de matéria orgânica ao

Tabela 4. Produção de $\mathrm{CO}_{2}$ acumulada aos 5, 15, 25, 35 e 45 dias de incubação nas profundidades $0-10 \mathrm{~cm}$ e 10-20 cm do solo para cada cobertura florestal estudada (floresta secundária, Acacia mangium, Sapindus saponaria e Hevea brasiliensis) na região sul do Espírito Santo.

Table 4. Cumulative $\mathrm{CO}_{2}$ production at $5,15,25,35$ and 45 days of incubation at depths of 0 to 10 and $10 \mathrm{~cm}$ to $20 \mathrm{~cm}$ for each studied forest cover (Forest secondary Acacia mangium, Sapindus saponaria and Hevea brasiliensis) in the south of Espírito Santo.

\section{Cobertura florestal}

\section{Produção acumulada de $\mathrm{CO}_{2}$}

\begin{tabular}{|c|c|c|c|c|c|}
\hline & 5 dias & 15 dias & 25 dias & 35 dias & 45 dias \\
\hline & \multicolumn{5}{|c|}{ 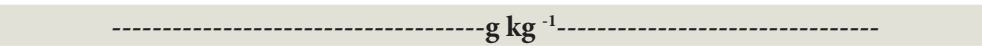 } \\
\hline \multicolumn{6}{|c|}{------------------------------- 0-10 cm ------------------------------ } \\
\hline Floresta secundária & 1,11 & 2,29 & 3,18 & 3,67 & 4,63 \\
\hline Sapindus saponaria & 1,58 & 3,45 & 4,53 & 5,12 & 6,29 \\
\hline Acacia mangium & 0,83 & 1,95 & 2,58 & 2,92 & 3,67 \\
\hline Hevea brasiliensis & 1,08 & 2,26 & 2,90 & 3,25 & 3,93 \\
\hline \multicolumn{6}{|c|}{ 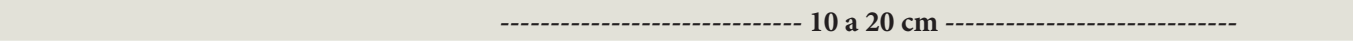 } \\
\hline Floresta secundária & 0,98 & 2,16 & 2,98 & 3,43 & 4,33 \\
\hline Sapindus saponaria & 1,94 & 4,03 & 5,43 & 6,12 & 7,40 \\
\hline Acacia mangium & 0,67 & 1,72 & 2,40 & 2,78 & 3,59 \\
\hline Hevea brasiliensis & 1,02 & 2,33 & 3,05 & 3,42 & 4,21 \\
\hline
\end{tabular}


solo promove estimulação da atividade microbiana, aumentando, consequentemente, a evolução de $\mathrm{CO}_{2}$. Outro aspecto importante a ser ressaltado, é o fato de que no presente estudo a serapilheira das coberturas florestais, antes de serem incorporadas ao solo para a montagem do experimento, foram moídas e passadas em peneira de $2 \mathrm{~mm}$. Esse fato aumenta a superfície da serapilheira que será decomposta, facilitando assim a ação dos microrganismos.

A curva de evolução de $\mathrm{CO}_{2}$ (produção acumulada de $\mathrm{CO}_{2}$ ) com o tempo de incubação foram ajustados na equação logística (Tabela 6). Na profundidade $0-10 \mathrm{~cm}$, a serapilheira da Acacia mangium apresentou o maior valor para o coeficiente $a$, indicando que essa apresenta características que favorecem sua decomposição e/ou maior proporção de carbono facilmente mineralizável.

Tabela 5. Contrastes das coberturas florestais nas profundidades $0-10 \mathrm{~cm}$ e $10-20 \mathrm{~cm}$ para a produção acumulada de $\mathrm{CO}_{2}$, aos 45 dias.

Table 5. Contrasts of forest cover depths $0-10 \mathrm{~cm}$ and $10-20 \mathrm{~cm}$ for the cumulative production of $\mathrm{CO} 2$, 45 days.

\begin{tabular}{ccc|}
\multirow{2}{*}{ Contraste } & \multicolumn{2}{c|}{ Profundidade } \\
\cline { 2 - 3 } & $\mathbf{0 - 1 0}(\mathbf{c m})$ & $\mathbf{1 0} \mathbf{~} \mathbf{2 0}(\mathbf{c m})$ \\
\hline C1 & $0,007^{\text {ns }}$ & $2,200^{\text {ns }}$ \\
C2 & $4,993^{\star *}$ & $7,010^{\text {** }}$ \\
\hline C3 & $2,107^{\text {ns }}$ & $2,560^{\text {ns }}$ \\
\hline
\end{tabular}

$\mathrm{C} 1$ = floresta secundária vs. acácia + saboneteira + seringueira; $\mathrm{C} 2$ = saboneteira vs. acácia + seringueira; $\mathrm{C} 3=$ seringueira vs acácia + saboneteira; NS = não significativo; ${ }^{* *}$ significativo a $1 \%$ de probabilidade.
Porém os resultados encontrados na decomposição da serapilheira dessa espécie mostram que ela possui o maior tempo de decomposição de sua serapilheira, juntamente com a seringueira (Tabela 2).

Já a serapilheira encontrada na floresta secundária apresentou o menor valor para o coeficiente $a$, indicando que essa possui características menos favoráveis para sua decomposição. Esse resultado era esperado, como discutido por Bini et al. (2013) a mistura de resíduos vegetais desfavorece a taxa de decomposição do material. O mesmo comportamento foi observado na profundidade $10-20 \mathrm{~cm}$. Observa-se que não houve grande variação nos valores encontrados para o coeficiente $b$, indicando um comportamento semelhante em todas as coberturas florestais.

Por meio da variável $t_{1 / 2}$ é possível estimar o tempo necessário para se atingir a metade da produção máxima de $\mathrm{CO}_{2}$. Essa variável está relacionada com a velocidade de mineralização da serapilheira, ou seja, maiores valores de $t_{1 / 2}$ indicam menores velocidades de mineralização e, portanto, menor labilidade do material.

Na profundidade $0-10 \mathrm{~cm}$, o maior valor de $t_{1 / 2}$ foi encontrado na serapilheira de saboneteira, indicando, assim, que essa possui menor velocidade de mineralização. Isso pode ser explicado pelo fato de essa espécie possuir em sua composição química a substância saponina, que tem propriedades detergentes e surfactantes, além de ser antifúngica, o que pode dificultar a ação de microrganismos, promovendo inativação dos escleródios (Monteiro, 2010). Porém, como visto anteriormente, a serapilheira de saboneteira apresentou maior produção

Tabela 6. Coeficientes da equação logística, coeficientes de determinação e tempo estimado para atingir a metade da produção máxima de $\mathrm{CO}_{2}\left(\mathrm{t}_{1 / 2}=-\mathrm{b} / \mathrm{c}\right)$ para cada cobertura florestal (floresta secundária, Acacia mangium, Sapindus saponaria e Hevea brasiliensis) na região sul do Espírito Santo.

Table 6. Coefficients of the logistic equation, coefficients of determination and estimated time to reach half the maximum $\mathrm{CO}_{2}\left(\mathrm{t}_{1 / 2}=-\mathrm{b} / \mathrm{c}\right)$ for each forest cover (Secondary forest, Acacia mangium, Sapindus saponaria and Hevea brasiliensis) in the south of Espírito Santo.

\begin{tabular}{|c|c|c|c|c|c|}
\hline Coberturas florestais & $a$ & $b$ & $c$ & $\mathbf{R}^{2}$ & $t_{1 / 2}$ (dias) \\
\hline \multicolumn{6}{|c|}{-----'- } \\
\hline Floresta secundária & 3,49 & $-1,88$ & 0,12 & 0,97 & 15,66 \\
\hline Sapindus saponaria & 4,61 & $-1,81$ & 0,11 & 0,98 & 16,45 \\
\hline Acacia mangium & 6,01 & $-1,72$ & 0,12 & 0,97 & 14,33 \\
\hline Hevea brasiliensis & 3,74 & $-1,64$ & 0,13 & 0,97 & 12,61 \\
\hline \multicolumn{6}{|c|}{ 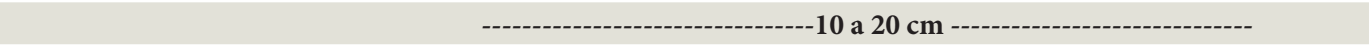 } \\
\hline Floresta secundária & 3,66 & $-2,16$ & 0,13 & 0,98 & 16,61 \\
\hline Sapindus saponaria & 4,38 & $-1,96$ & 0,12 & 0,98 & 16,33 \\
\hline Acacia mangium & 7,09 & $-1,73$ & 0,12 & 0,98 & 14,41 \\
\hline Hevea brasiliensis & 4,16 & $-1,89$ & 0,13 & 0,98 & 14,53 \\
\hline
\end{tabular}


acumulada de $\mathrm{CO}_{2}$, diferenciando-se significativamente em relação às outras espécies.

Para a mesma profundidade $(0-10 \mathrm{~cm})$, o menor valor de $t_{1 / 2}$ foi observado para a espécie seringueira, indicando que sua serapilheira possui maior velocidade de mineralização. Uma possível justificativa é o material vegetal dessa espécie apresentar-se mais "fino" quando comparado ao das outras espécies, o que pode facilitar sua decomposição. Esse resultado não era esperado, já que essa espécie possui vasos lactíferos que podem tornar o material vegetal menos palatável para os microrganismos.

Na profundidade $10-20 \mathrm{~cm}$ do solo, os resultados encontrados para as coberturas florestais seguiram o mesmo comportamento para a profundidade 0-10 cm, ou seja, a saboneteira e a floresta secundária apresentaram maiores valores para $t_{1 / 2}$, indicando para essas espécies uma velocidade de decomposição da serapilheira mais lenta.

\section{CONCLUSÕES}

Dentre as serapilheiras das coberturas florestais avaliadas, a de Sapindus saponaria apresentou maior velocidade de decomposição e a de Hevea brasiliensis a menor.

Os teores de nutrientes liberados por meio da decomposição da serapilheira das coberturas florestais apresentaram-se, de maneira geral, decrescentes com o tempo.

A cobertura de Sapindus saponaria apresentou, para as duas profundidades do solo, quantidades acumuladas de $\mathrm{CO}_{2}$ superiores em relação às outras coberturas florestais.

\section{STATUS DA SUBMISSÃO}

Recebido: 20 mar., 2016

Aceito: 28 ago., 2016

\section{AUTOR(ES) PARA CORRESPONDÊNCIA}

\section{Felipe Vaz Andrade}

Departamento de Produção Vegetal, Universidade Federal do Espirito Santo - UFES, Alto Universitário, s/n, Guararema, CP 16, CEP 29500-000, Alegre, ES, Brasil e-mail: felipe.andrade@ufes.br

\section{REFERENNCIAS}

Anderson JPE. Soil respiration. In: Page AL, Miller RH, Keeney DR, editores. Methods of soil analysis. Part 2. 2nd ed. Madison: American Society of Agronomy, Soil Science Society of America; 1982. p. 831-866.

Andrade FV, Mendonça ES, Silva IRD. Organic acid adsorption and mineralization in oxisols with different textures. Revista Brasileira de Ciencia do Solo 2013; 37(4): 976-985. http://dx.doi.org/10.1590/S0100-06832013000400015.

Balieiro FC, Dias LE, Campello EFC, Faria SM. Acúmulo de nutrientes na parte aérea, na serapilheira acumulada sobre o solo e decomposição de filódios de Acacia mangium Willd. Ciência Florestal 2004; 14(1): 59-65.

Barros NF, Comerford NB. Sustentabilidade da produção de florestas plantadas na região tropical. In: Alvarez VVH, Schaefer CEGR, Barros NF, Mello JWV, Costa LM, editores. Tópicos em ciência do solo. Viçosa: Sociedade Brasileira Ciência do Solo; 2002. p. 487-592.

Bini D, Figueiredo AF, Silva MCP, Vasconcellos RLF, Cardoso EJBN. Microbial biomass and activity in litter during the initial development of pure and mixed plantations of Eucalyptus grandis and Acacia mangium. Revista Brasileira de Ciencia do Solo 2013; 37(1): 76-85. http://dx.doi.org/10.1590/S0100-06832013000100008.

Carvalho AM, Bustamante MMC, Sousa JGA Jr, Vivaldi LJ. Decomposição de resíduos vegetais em latossolo sob cultivo de milho e plantas de cobertura. Revista Brasileira de Ciencia do Solo 2008; 32(spe): 2831-2838. http://dx.doi. org/10.1590/S0100-06832008000700029.

Cianciaruso MV, Pires JSR, Delitti WBC, Silva EFLP. Produção de serapilheira e decomposição do material foliar em um cerradão na Estação Ecológica de Jataí, município de Luiz Antônio, SP, Brasil. Acta bot. Brás 2006; 20(1): 49-59.

Cobo JG, Barrios E, Kass DCL, Thomas RJ. Nitrogen mineralization and crop uptake from surface-applied leaves of green manure on a tropical volcanic-ash soil. Biology and Fertility of Soils 2002; 36(2): 87-92. http:// dx.doi.org/10.1007/s00374-002-0496-y.

Colle DW, Rapp M. Elemental cycling in forested ecosystems. In: Reichle DE, editor. Dynamic properties of forest ecosystems. Cambridge: Cambridge University; 1980. p. 341-409.

Cornu S, Luizão F, Rouiller J, Lucas Y. Comparative study of litter decomposition and mineral element release in two Amazonian Forest ecosystems: litter bag experiments. Pedobiologia 1997; 41: 456-471.

Correia MEF, Andrade AG. Formação da serrapilheira e ciclagem de nutrientes. In: Santos GA, Camargo FAO, editores. Fundamentos da matéria orgânica do solo ecossistemas tropicais e subtropicais. Porto Alegre: Genesis; 1999. p. 197-225. 
Cunha FV No, Leles PSS, Pereira MG, Bellumath VGH, Alonso JM. Acúmulo e decomposição da serapilheira em quatro formações florestais. Ciência Florestal 2013; 23(3): 379-387. http://dx.doi.org/10.5902/1980509810549.

Dickinson C. Decomposition of litter in soil. In: Dickinson, C, Pugh, G, editores. Biological of plant litter decomposition. San Diego: Academic Press; 1974. p. 633-654. vol. 2.

Empresa Brasileira de Pesquisa Agropecuária - EMBRAPA. Centro Nacional de Pesquisa de Solos. Manual de métodos de análise de solo. Rio de Janeiro: EMBRAPA; 1997.

Empresa Brasileira de Pesquisa Agropecuária - EMBRAPA. Centro Nacional de Pesquisa de Solos. Sistema brasileiro de classificação de solos. Brasília: Produção de Informação; Rio de Janeiro: Embrapa Solos; 1999.

Fernandes MM, Pereira MG, Magalhães LMS, Cruz AR, Giácomo RG. Aporte e decomposição de serapilheira em áreas de floresta secundária, plantio de sabiá (Mimosa caesalpiniaefolia Benth.) e andiroba (Carapa guianensis Aubl.) na Flona Mário Xavier, RJ. Ciência Florestal 2006; 16(2): 163-175. http://dx.doi.org/10.5902/198050981897.

Gama-Rodrigues AC, Barros NF, Santos ML. Decomposição e liberação de nutrientes do folhedo de espécies florestais nativas em plantios puros e mistos no sudeste da Bahia. Revista Brasileira de Ciencia do Solo 2003; 27(6): 1021-1031. http://dx.doi.org/10.1590/S0100-06832003000600006.

Gama-Rodrigues AC, Gama-Rodrigues EF, Barros NF. Balanço de carbono e nutrientes em plantio puro e misto de espécies florestais nativas no sudeste da Bahia. Revista Brasileira de Ciencia do Solo 2008; 32(5): 1165-1179. http://dx.doi.org/10.1590/S0100-06832008000300025.

Godinho TO, Caldeira MVW, Rocha JHT, Caliman JP, Trazzi PQ. Quantificação de biomassa e nutrientes na serapilheira acumulada em trecho de floresta estadual semidecidual submontana, ES. Cerne 2014; 20(1): 11-20. http://dx.doi.org/10.1590/S0104-77602014000100002.

Gonçalves SL, Saraiva OF, Torres E. Influência de fatores climáticos na decomposição de resíduos culturais de milho e soja. Londrina: Embrapa Soja; 2011. 25 p. Boletim de Pesquisa e Desenvolvimento.

Inknotte J, Mafra AL, Rios PD, Baretta D, Vieira HC. Deposição de serapilheira em reflorestamentos de eucalipto e florestas nativas nas regiões Planalto e Oeste do Estado de Santa Catarina. Scientia Forestalis 2015; 43(106): 261-270.

Instituto Capixaba de Pesquisa, Assistência Técnica e Extensão Rural - INCAPER. Programa de Assistência Técnica e Extensão Rural 2010 [online]. Vitória: INCAPER; 2010. [citado em 2011 mar. 1]. Disponível em: http:// www.incaper.es.gov.br/proater/

Jensen V. Decomposition of angiosperm tree leaf litter. In: Dickinson C, Pugh G, editores. Biological of plant litter decomposition. San Diego: Academic Press; 1974. p. 69-104. vol. 2.
Loss A, Pereira MG, Beutler SJ, Perin A, Anjos LHC. Carbono mineralizável, carbono orgânico e nitrogênio em macroagregados de Latossolo sob diferentes sistemas de uso do solo no Cerrado Goiano. Ciências Agrárias 2013; 34(5): 2153-2168.

Lupwayi NZ, Clayton GW, O’Donovan JT, Harker KN, Turkington TK, Rice WA. Decomposition of crop residues under conventional and zero tillage. Journal of Soil Science 2004;(84): 403-410.

Mercante FM, Frabricio AC, Machado LAZ, Silva WM. Parâmetros microbiológicos como indicadores de qualidade do solo sob sistema integrados de produção agropecuária. Dourados: Embrapa Agropecuária Oeste; 2004. 27 p. Boletim de Pesquisa e Desenvolvimento v. 20.

Monteiro FP. Interferência de plantas de cobertura no comportamento de Sclerotina sclerotiorum [dissertação]. Lavras: Universidade Federal de Lavras; 2010.

Moreira FMS, Siqueira JO. Microbiologia e bioquímica do solo. 2. ed. Lavras: UFLA; 2006. 729 p.

Oliveira RB. Mapeamento e correlação de atributos do solo e de plantas de café conilon para fins de agricultura de precisão [dissertação]. Alegre: Universidade Federal do Espírito Santo; 2007.

Passos RR, Ruiz HA, Mendonça ES, Cantarutti RB, Souza AP. Substâncias húmicas, atividade microbiana e carbono orgânico lábil em agregados de um Latossolo vermelho distrófico sob duas coberturas vegetais. Revista Brasileira de Ciencia do Solo 2007; 31(5): 1119-1129. http://dx.doi. org/10.1590/S0100-06832007000500027.

Pegado CMA, Barbosa LJN, Mendes JEMF, Souto PC, Souto JC. Decomposição superficial e subsuperficial de folhas de fava (Phaseolus lunatus L.) na região do Brejo da Paraiba, Brasil. Caatinga 2008; (21): 218-223.

Santana JAS, Souto JS. Produção de serapilheira na Caatinga da região semi-árida do Rio Grande do Norte, Brasil. Idesia 2011; 29(2): 87-94. http://dx.doi.org/10.4067/ S0718-34292011000200011.

Selle GL. Ciclagem de nutrientes em ecossistemas florestais. Bioscience Journal 2007; 23(4): 29-39.

Silva AG, Gonçalves MAM, Reis EF. Decomposição e teor de nutrientes da serapilheira foliar em um fragmento de Floresta Atlântica no sul do estado e do Espírito Santo. Ecologia Nutrição Florestal 2013; 1(2): 63-71. http://dx.doi. org/10.13086/2316-980x.v01n02a02.

Silva HF, Barreto PAB, Sousa GTO, Azevedo GB, GamaRodrigues EF, Oliveira FGRB. Decomposição de serapilheira foliar em três sistemas florestais no Sudoeste da Bahia. Revista Brasileira de Biociências 2014; 12(3): 164-172.

Smith VC, Bradford MA. Litter quality impacts on grassland litter decomposition are differently dependent on soil fauna across time. Applied Soil Ecology, 2003; 24(2): 197-203. 
Souto PC. Acumulação e decomposição da serapilheira e distribuição de organismos edáficos em área de caatinga na Paraíba, Brasil [tese]. Areia: Universidade Federal da Paraíba; 2006.

Swift MJ, Heal OW, Anderson JM. Decomposition in terrestrial ecosystems. Berkeley: University of California Press; 1979. 372 p.

Taiz L, Zeiger E. Fisiologia vegetal. 4. ed. Artmed: Porto Alegre; 2009.

Tedesco MJ, Gianello C, Bissani CA, Bohnen H, Volkweiss SJ. Análise de solo, plantas e outros materiais. Porto Alegre: UFRGS; 1995. 174 p.

Thomas RJ, Asakawa NM. Decomposition of leaf litter from tropical forage grasses and legumes. Soil Biology \& Biochemistry 1993; 25(10): 1351-1361. http://dx.doi. org/10.1016/0038-0717(93)90050-L.
Valente FD, Neves LG, Tienne L, Marques O, Cortines E, Valcarcel R. Produção e decomposição de serapilheira em medidas biológicas de reabilitação de áreas de empréstimo na Mata Atlântica. Revista Universidade Rural 2005; 25(1): 18-25.

Vieira M, Schumacher MV, Araújo EF. Floresta e Ambiente 2014; 21(3): 307-315. http://dx.doi.org/10.1590/21798087.066313 .

Waring RH, Schlesinger WH. Decomposition and forest soil development. In: Waring RH, Schlesinger WH. Forest ecosystems: concept and management. New York: Academic Press; 1985. p. 340-365.

Yeomans JC, Bremner JM. A rapid and precise method for routine determination of organic carbon in soil. Communications in Soil Science and Plant Analysis 1988, 19(1): 1467-1476. 